\title{
Haematological parameters and anaemia in white and British Indian meat-eaters and vegetarians in UK Biobank
}

\author{
T.Y.N. Tong ${ }^{1}$, T.J. Key ${ }^{1}$, K. Gaitskell ${ }^{1}$, T.J. Green ${ }^{2}$, W. Guo ${ }^{1}$, T.A. Sanders ${ }^{3}$ and \\ K.E. Bradbury ${ }^{1,4}$ \\ ${ }^{1}$ Cancer Epidemiology Unit, Nuffield Department of Population Health, University of Oxford, OX3 7LF, \\ ${ }^{2}$ Discipline of Paediatrics and Reproductive Health, School of Medicine, University of Adelaide, Australia, \\ ${ }^{3}$ Department of Nutritional Sciences, King's College London, WC2R $2 L S$ and \\ ${ }^{4}$ School of Population Health, Faculty of Medical and Health Sciences, University of Auckland, New Zealand
}

There may be differences in haematological parameters between meat-eaters and vegetarians ${ }^{(1,2)}$. We aimed to perform cross-sectional analyses of haematological parameters by diet group in a large United Kingdom (UK) cohort.

A complete blood count was carried out in all UK Biobank participants at recruitment $(2006-2010)^{(3)}$. We examined haemoglobin, red and white blood cell counts, and platelet counts and volume, in regular meat-eaters ( $>3$ times/week of red/processed meat consumption, $n=212,861)$, low meat-eaters $(n=213,119)$, poultry-eaters $(n=4,816)$, fish-eaters $(n=10,044)$, vegetarians $(n=6,550)$, and vegans $(n=398)$ of white ethnicity, and meat-eaters $(n=3,875)$ and vegetarians $(n=1,362)$ of British Indian ethnicity.

In both white and British Indian populations, compared with regular meat-eaters (or all meat-eaters among Indians), the other diet groups had up to $3.7 \%$ lower age adjusted haemoglobin concentrations (difference not significant in white vegan women), and were generally more likely to have anaemia (e.g. $8.7 \%$ of regular meat-eaters versus $12.7 \%$ of vegetarians in white premenopausal women). In the white population, compared with regular meat-eaters, all other diet groups had lower age and sex adjusted total white cells, neutrophils, lymphocytes, monocytes and eosinophils (p-heterogeneity $<0.001$ for all), but basophil counts were similar across diet groups; in British Indians, there was no significant difference in any of the white cell counts by diet group. Compared with white regular meat-eaters, the low meat-eaters, poultry-eaters, fish-eaters, and vegans had lower platelet counts and higher platelet volume, while vegetarians had higher counts and lower volume; compared with British Indian meat-eaters, vegetarians had higher platelet count and lower volume.

In UK Biobank, people with low or no red meat intake generally had lower haemoglobin concentrations and were slightly more likely to be anaemic. The lower white cell counts observed in low and non-meat eaters, and differences in mean platelet counts and volume between diet groups, warrant further investigation.
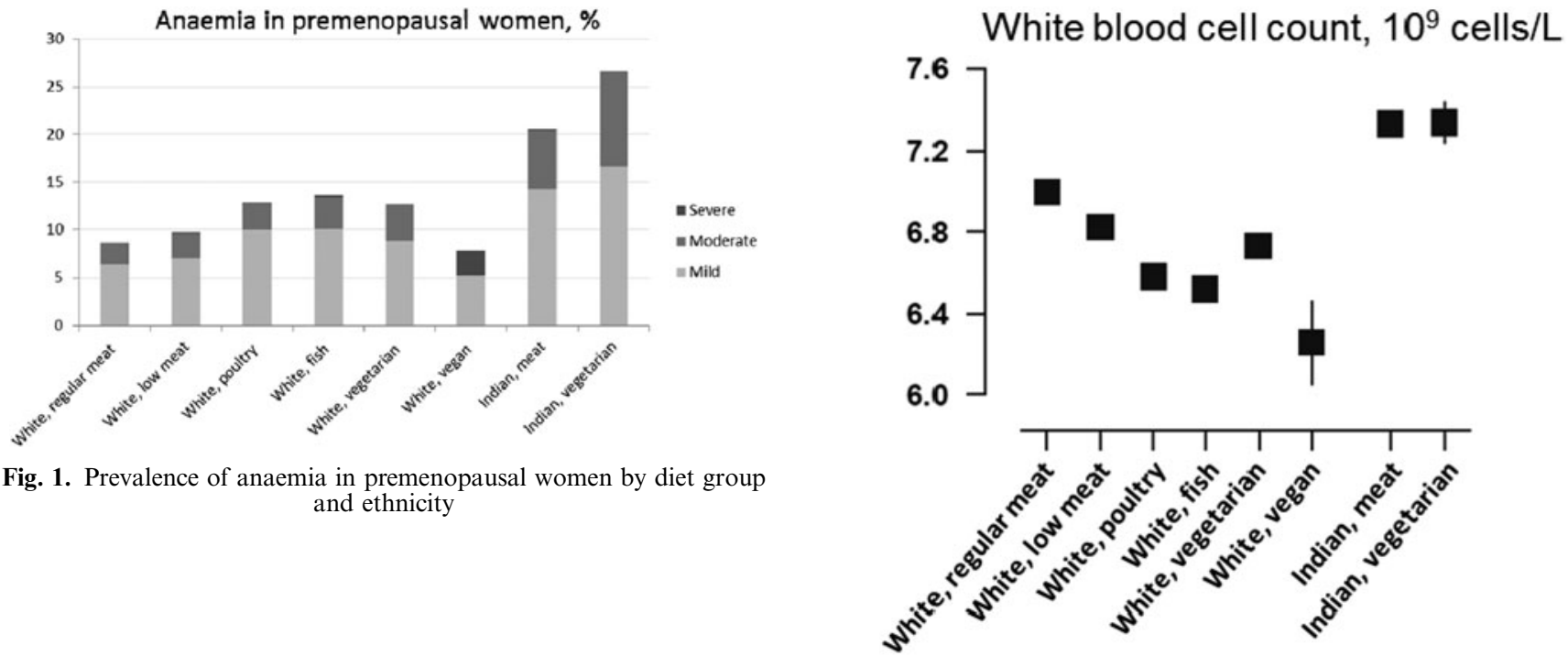

Fig. 2. White blood cell count by diet group and ethnicity

This work was supported by the UK Medical Research Council (MR/M012190/1) and the Wellcome Trust Our Planet Our Health (Livestock, Environment and People, LEAP 205212/Z/16/Z). KEB was supported by the Girdlers' New Zealand Health Research Council Fellowship.

1. Reddy S \& Sanders TA (1990) Br J Nutr 64, 331-8.

2. Haddad EH, Berk LS, Kettering JD, et al. (1999) Am J Clin Nutr 70(SUPPL)., 586S-93S

3. Elliott P \& Peakman TC (2008) Int J Epidemiol 37, 234-44. 